DOI: $10.21105 /$ joss. 02018

\title{
Oceananigans.jl: Fast and friendly geophysical fluid dynamics on GPUs
}

\author{
Ali Ramadhan ${ }^{1}$, Gregory LeClaire Wagner ${ }^{1}$, Chris Hill ${ }^{1}$, Jean-Michel \\ Campin $^{1}$, Valentin Churavy ${ }^{1}$, Tim Besard ${ }^{2}$, Andre Souza ${ }^{1}$, Alan \\ Edelman $^{1}$, Raffaele Ferrari ${ }^{1}$, and John Marshall ${ }^{1}$
}

1 Massachusetts Institute of Technology 2 Julia Computing, Inc.

\section{Software}

- Review ¿

- Repository ¿

- Archive c

Editor: Kristen Thyng Ø Reviewers:

- @funsim

- Omancellin

Submitted: 17 December 2019 Published: 22 September 2020

\section{Summary}

Oceananigans. $j 1$ is a fast and friendly software package for the numerical simulation of incompressible, stratified, rotating fluid flows on CPUs and GPUs. Oceananigans. $j 1$ is fast and flexible enough for research yet simple enough for students and first-time programmers. Oceananigans. $\mathrm{jl}$ is being developed as part of the Climate Modeling Alliance project for the simulation of small-scale ocean physics at high-resolution that affect the evolution of Earth's climate.

Oceananigans.jl is designed for high-resolution simulations in idealized geometries and supports direct numerical simulation, large eddy simulation, arbitrary numbers of active and passive tracers, and linear and nonlinear equations of state for seawater. Under the hood, Oc eananigans. $\mathrm{jl}$ employs a finite volume algorithm similar to that used by the Massachusetts Institute of Technology general circulation model (Marshall, Adcroft, Hill, Perelman, \& Heisey, 1997).

Authors of papers retain copyright and release the work under a Creative Commons Attribution 4.0 International License (CC BY 4.0).

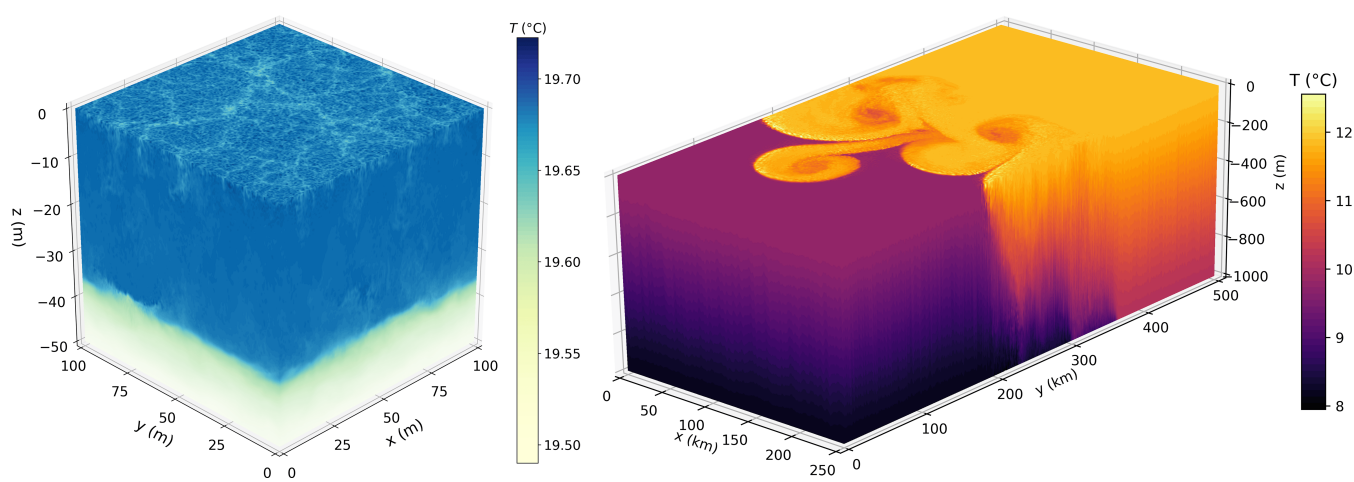

Fig. 1: (Left) Large eddy simulation of small-scale oceanic boundary layer turbulence forced by a surface cooling in a horizontally periodic domain using $256^{3}$ grid points. The upper layer is well-mixed by turbulent convection and bounded below by a strong buoyancy interface. (Right) Simulation of instability of a horizontal density gradient in a rotating channel using $256 \times 512 \times 128$ grid points. A similar process called baroclinic instability acting on basin-scale temperature gradients fills the oceans with eddies that stir carbon and heat. Plots made with matplotlib (Hunter, 2007) and cmocean (Thyng, Greene, Hetland, Zimmerle, \& DiMarco, 2016).

Oceananigans . jl leverages the Julia programming language (Bezanson, Edelman, Karpinski, \& Shah, 2017) to implement high-level, low-cost abstractions, a friendly user interface, and a high-performance model in one language and a common code base for execution on the CPU or GPU with Julia's native GPU compiler (Besard, Foket, \& De Sutter, 2019). Because 
Julia is a high-level language, development is streamlined and users can flexibly specify model configurations, set up arbitrary diagnostics and output, extend the code base, and implement new features. Configuring a model with architecture=CPU() or architecture=GPU() will execute the model on the CPU or GPU. By pinning a simulation script against a specific version of Oceananigans, simulation results are reproducible up to hardware differences.

Performance benchmarks show significant speedups when running on a GPU. Large simulations on an Nvidia Tesla V100 GPU require $\sim 1$ nanosecond per grid point per iteration. GPU simulations are therefore roughly $3 x$ more cost-effective than CPU simulations on cloud computing platforms such as Google Cloud. A GPU with 32 GB of memory can time-step models with $\sim 150$ million grid points assuming five fields are being evolved; for example, three velocity components and tracers for temperature and salinity. These performance gains permit the long-time integration of realistic simulations, such as large eddy simulation of oceanic boundary layer turbulence over a seasonal cycle or the generation of training data for turbulence parameterizations in Earth system models.

Oceananigans. $j 1$ is continuously tested on CPUs and GPUs with unit tests, integration tests, analytic solutions to the incompressible Navier-Stokes equations, convergence tests, and verification experiments against published scientific results. Future development plans include support for distributed parallelism with CUDA-aware MPI as well as topography.

Ocean models that are similar to Oceananigans.jl include MITgcm (Marshall et al., 1997) and MOM6 (Adcroft et al., 2019), both written in Fortran. However, Oceananigans.j1 features a more efficient non-hydrostatic pressure solver than MITgcm (and MOM6 is strictly hydrostatic). PALM (Maronga et al., 2020) is Fortran software for large eddy simulation of atmospheric and oceanic boundary layers with complex boundaries on parallel CPU and GPU architectures. Oceananigans. $\mathrm{jl}$ is distinguished by its use of Julia which allows for a scriptbased interface as opposed to a configuration-file-based interface used by MITgcm, MOM6, and PALM. Dedalus (Burns, Vasil, Oishi, Lecoanet, \& Brown, 2020) is Python software with an intuitive script-based interface that solves general partial differential equations, including the incompressible Navier-Stokes equations, with spectral methods.

\section{Acknowledgements}

Our work is supported by the generosity of Eric and Wendy Schmidt by recommendation of the Schmidt Futures program, and by the National Science Foundation under grant AGS-6939393.

\section{References}

Adcroft, A., Anderson, W., Balaji, V., Blanton, C., Bushuk, M., Dufour, C. O., Dunne, J. P., et al. (2019). The GFDL Global Ocean and Sea Ice Model OM4.0: Model Description and Simulation Features. Journal of Advances in Modeling Earth Systems, 11(10), 3167-3211. doi:10.1029/2019MS001726

Besard, T., Foket, C., \& De Sutter, B. (2019). Effective Extensible Programming: Unleashing Julia on GPUs. IEEE Transactions on Parallel and Distributed Systems, 30(4), 827-841. doi:10.1109/TPDS.2018.2872064

Bezanson, J., Edelman, A., Karpinski, S., \& Shah, V. B. (2017). Julia: A Fresh Approach to Numerical Computing. SIAM Review, 59(1), 65-98. doi:10.1137/141000671

Burns, K. J., Vasil, G. M., Oishi, J. S., Lecoanet, D., \& Brown, B. P. (2020). Dedalus: A flexible framework for numerical simulations with spectral methods. Phys. Rev. Research, 2(2), 023068. doi:10.1103/PhysRevResearch.2.023068 
Hunter, J. D. (2007). Matplotlib: A 2D graphics environment. Computing in Science \& Engineering, 9(3), 90-95. doi:10.1109/MCSE.2007.55

Maronga, B., Banzhaf, S., Burmeister, C., Esch, T., Forkel, R., Fröhlich, D., Fuka, V., et al. (2020). Overview of the PALM model system 6.0. Geoscientific Model Development, 13(3), 1335-1372. doi:10.5194/gmd-13-1335-2020

Marshall, J., Adcroft, A., Hill, C., Perelman, L., \& Heisey, C. (1997). A finite-volume, incompressible Navier Stokes model for studies of the ocean on parallel computers. Journal of Geophysical Research: Oceans, 102(C3), 5753-5766. doi:10.1029/96JC02775

Thyng, K. M., Greene, C. A., Hetland, R. D., Zimmerle, H. M., \& DiMarco, S. F. (2016). True Colors of Oceanography: Guidelines for Effective and Accurate Colormap Selection. Oceanography, 29(3), 9-13. doi:10.5670/oceanog.2016.66 